\title{
Accurately Quantifying Clear-Sky Radiative Cooling Potentials: A Temperature Correction to the Transmittance-Based Approximation
}

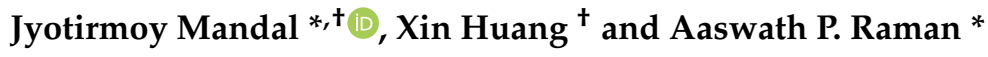 \\ Department of Materials Science and Engineering, University of California, Los Angeles, CA 90024, USA; \\ uclahuangxin@g.ucla.edu \\ * Correspondence: jyotirmoymandal@g.ucla.edu (J.M.); aaswath@ucla.edu (A.P.R.) \\ + These authors contributed equally to the work.
}

Citation: Mandal, J.; Huang, X.; Raman, A.P. Accurately Quantifying Clear-Sky Radiative Cooling Potentials: A Temperature Correction to the Transmittance-Based Approximation. Atmosphere 2021, 12, 1195. https:// doi.org/10.3390/atmos12091195

Academic Editors: Giulia Ulpiani, Chi-Yan Tso and Yu-Bin Chen

Received: 17 August 2021

Accepted: 13 September 2021

Published: 15 September 2021

Publisher's Note: MDPI stays neutral with regard to jurisdictional claims in published maps and institutional affiliations.

Copyright: (C) 2021 by the authors Licensee MDPI, Basel, Switzerland. This article is an open access article distributed under the terms and conditions of the Creative Commons Attribution (CC BY) license (https:/ / creativecommons.org/licenses/by/ $4.0 /)$.

\begin{abstract}
Theoretical calculations of the cooling potential of radiative cooling materials are crucial for determining their cooling capability under different meteorological conditions and evaluating their performance. To facilitate these calculations, accurate models of long-wave infrared downwelling atmospheric irradiance are needed. However, the transmittance-based cosine approximation, which is widely used to determine radiative cooling potentials under clear sky conditions, does not account for the cooling potential arising from heat loss to the colder reaches of the atmosphere itself. Here, we show that use of the approximation can lead to $>10 \%$ underestimation of the cooling potential relative to MODTRAN 6 outputs. We propose a temperature correction to the transmittance-based approximation, which accounts for heat loss to the cold upper atmosphere, and significantly reduces this underestimation, while retaining the advantages of the original model. In light of the widespread and continued use of the transmittance-based model, our results highlight an important source of potential errors in the calculation of clear sky radiative cooling potentials and a means to correct for them.
\end{abstract}

Keywords: radiative cooling; cooling potential; MODTRAN; transmittance model; atmospheric optics; atmospheric irradiance

\section{Introduction}

In recent years, radiative cooling has seen growing scientific and commercial interest for applications ranging from the passive cooling of buildings to geoengineering. The process, which involves a spontaneous heat loss from terrestrial objects to the atmosphere and outer space by radiation of heat (and reflection of incident sunlight) through atmospheric transmission windows, has a zero-energy, zero-carbon functionality and a net cooling effect on the environment [1]. Precisely how much cooling occurs for a given surface depends strongly on meteorological conditions. For instance, the cooling potential, which is defined as the difference between the radiance from a sky-facing radiative cooler and the downwelling atmospheric irradiance under clear skies, can vary between $\sim 0$ and $150 \mathrm{Wm}^{-2}$ depending on the ambient temperature and total precipitable water (TPW) content [2].

Given this large variability, accurate determination of radiative cooling potentials is crucial for validating the performance of radiative coolers, informing industry on the geographical scope of designs such as cool-roof paints [3], and the best potential geographic regions for radiative cooling approaches for geoengineering [2,4]. Research over the years has yielded a range of atmospheric models that can be used to calculate radiative cooling potentials, accounting for various factors such as humidity, topography, cloud cover and altitude [1,5-12]. However, standard models used today to calculate radiative cooling potentials or cooling power are often used beyond their scope, leading to systematic errors. The most prevalent example of this is the transmittance-based cosine approximation. This 
approximation is widely used to model radiative cooling potentials under clear skies, particularly of materials with strong spectral and angular selectivity in their emissivity.

In this paper, we elucidate the source of the errors in atmospheric radiance calculations that use the transmittance-based cosine approximation and demonstrate its underestimation of radiative cooling potential due to its simplified accounting of the irradiance from greenhouse gases where the atmosphere is transparent. Comparative analysis against the MODTRAN atmospheric hemispherical irradiance model shows that the transmittancebased cosine approximation yields a significantly higher downwelling atmospheric irradiance, and thus, cooling potentials that are lower by $6-24 \mathrm{Wm}^{-2}$, under typical operating conditions, which is $10-23 \%$ more than the approximation itself. To address this, we apply a temperature correction that accounts for the high elevations, and thus, low temperatures, of greenhouse gases, namely, water vapor, carbon dioxide and ozone, which allows a net heat transfer to them from the earth's surface. This reduces the underestimation of the cooling potential to $0.1-6 \%$ while retaining the useful angular resolution of the transmittance-based cosine approximation, which the MODTRAN hemispherical irradiance model does not provide. Our results suggest that recently constructed maps of radiative cooling potentials may require corrections. Moreover, they indicate that the common use of the uncorrected transmittance-based cosine approximation to verify experimental demonstrations of radiative cooling could be leading to an overestimation of performance of radiative cooling designs across the literature.

\section{Atmospheric Irradiance and the Transmittance-Based Cosine Approximation}

Due to its constituent greenhouse gases that are intrinsically absorptive or emissive in the thermal wavelengths, the atmosphere radiates heat towards the earth's surface. The difference between this irradiance $I_{a t m}$ and the black-body radiance $I_{B B}\left(T_{a m b}\right)$ at the ambient air temperature $\left(T_{a m b}\right)$ close to the ground is what is typically defined as the cooling potential or cooling power $P_{\text {cooling }}$. The cooling potential arises primarily within the long-wavelength infrared (LWIR, 8-13 $\mu \mathrm{m}$ ) atmospheric transmission window, where the low intrinsic absorption of water vapor lowers $I_{a t m}$, and reveals the cold upper reaches of the atmosphere and the cold space beyond, allowing for heat radiated upwards from the ground to be lost.

While the calculation of black-body irradiance is straightforward, it is more challenging to calculate $I_{a t m}$, which needs to, at the very least, account for the spectral properties of greenhouse gases, their distributions along the height of the atmosphere, and variations in temperature across heights. A long history of work on this topic has yielded a number of useful theoretical models for calculating $I_{a t m}$, each of which is reasonably accurate within its scope of use [1,5-9]. For instance, when using a spectrally flat emitter (grayor black-body), simple correlations have been shown to be very accurate in predicting downwelling atmospheric irradiance [6]. However, for many radiative cooling calculations, spectrally and angularly resolved sky irradiance is often required, since radiative cooling surfaces can present highly spectral and angular selective emissivities. Models that yield the level of detail needed for such calculations are comparatively rare $[1,5,13]$. One model, which has achieved almost universal use in recent radiative cooling literature, is the transmittance-based cosine approximation [1,14-29], which was first used as part of a more comprehensive model by Granqvist in 1981 [1]. This model assumes that the irradiance of the atmosphere originates from greenhouse gases, including water vapor, carbon dioxide and ozone, and calculates the spectral, angular sky irradiance based on an effective spectral angular emittance as follows:

$$
I_{a t m}\left(\theta, \lambda, T_{a t m}\right)=\varepsilon_{a t m}\left(\theta, \lambda, T_{a m b}\right) \cdot I_{B B}\left(\theta, \lambda, T_{a t m}\right)
$$

where

$$
\varepsilon_{a t m}\left(\theta, \lambda, T_{a m b}\right)=1-\left[1-\varepsilon_{a t m}\left(0, \lambda, T_{a m b}\right)\right]^{1 / \cos \theta}
$$


Here, $\varepsilon_{a t m}$ is the effective emittance of the atmosphere, $\theta$ is the angle measured from the zenith and $\lambda$ is the wavelength. As stated by Granqvist, implicit in this model is Kirchhoff's Law, which states that at thermal equilibrium:

$$
\varepsilon_{\text {atm }}(\theta, \lambda)=\alpha_{a t m}(\theta, \lambda)=1-\tau_{a t m}(\theta, \lambda)
$$

where $\alpha_{a t m}(\theta, \lambda)$ is the spectral, directional absorptance of the atmosphere. $\tau_{a t m}(\theta, \lambda)$ is the spectral, directional transmittance and is calculated based on the zenith-ward value $\tau_{\text {atm }}(0, \lambda)$ using the cosine approximation:

$$
\tau_{\text {atm }}(\theta, \lambda)=\tau_{\text {atm }}(0, \lambda)^{\frac{1}{\cos \theta}}
$$

The hemispherical irradiance $I_{a t m}$ can be calculated by hemispherical integration of the angular values from (1) and, in turn, yields $P_{\text {cooling }}=I_{B B}-I_{\text {atm }}$.

\section{Issues with the Transmittance-Based Cosine Approximation}

While the model provides a reasonable estimate of $I_{a t m}(\theta, \lambda)$, a crucial point to note is that in using Kirchhoff's law and Equation (3), it implicitly assumes that the atmosphere is a homogenous entity at thermal equilibrium [1]. In reality, it is a complex, semi-transparent structure whose temperature decreases with height. Where the atmosphere is particularly transparent, the colder upper reaches of the atmosphere are apparent from the ground. This transparency enables heat loss not only through the atmosphere but also to it (Figure 1A), a fact that is not accounted for by the transmittance-based approximation.

The case for ozone illustrates this well. It is first important to note that the overwhelming majority of ozone occurs at frigid 10-40 km heights of the stratosphere in what is known as the ozone layer. Consequently, any downwelling irradiance outside the LWIR window from ozone is masked by the highly absorptive water vapor and $\mathrm{CO}_{2}$ in the troposphere. In the LWIR window, however, the transparency of the atmosphere reveals the ozone layer and outer space beyond. This means that some of the radiance from the earth's surface is absorbed by the ozone, and much of its intrinsic radiance at $\sim 9.5 \mu \mathrm{m}$ reaches the earth. However, because of the low temperature of the ozone layer, which can be $\sim 70{ }^{\circ} \mathrm{C}$ lower than $T_{a m b}$ at altitudes where ozone concentration peaks in the summer and $\sim 40{ }^{\circ} \mathrm{C}$ lower in the winter [30], it radiates far less towards the ground than it absorbs from the latter. In other words, a net heat loss occurs from the surface to the ozone layer, and the assumption of a thermally homogeneous atmosphere no longer holds (Figure 1A). This is not captured by Equation (3), which implicitly assumes the ozone layer to be at $T_{a m b}$, causing the downwelling irradiance from ozone to be incorrectly equal to the fraction of $I_{B B}\left(T_{a m b}\right)$ absorbed by it. Consequently, heat loss to the ozone layer is not registered, $I_{\text {atm }}$ is overestimated and $P_{\text {cooling }}$ is underestimated, as is shown in Figure 1B. This is also evident when one compares the hemispherical emittance calculated by MODTRAN with $I_{\text {atm }}$ calculated using the transmittance-based cosine approximation. The difference due to the ozone effect alone is about $5-18.5 \mathrm{Wm}^{-2}$ depending on the atmosphere type and temperature, which is a significant $6-21 \%$ of the net cooling potential predicted by the transmittance-based cosine approximation.

A similar effect occurs for water vapor and carbon dioxide, which we consider collectively in this analysis. The two gases are well mixed throughout the atmosphere, and a majority of their downwelling irradiance arises from within $\sim 10^{2} \mathrm{~m}$ depths of the atmosphere near the earth's surface outside the LWIR window (which is at $\sim T_{a m b}$ ) and within $2 \mathrm{~km}$ of the earth's surface (or within $\sim 12{ }^{\circ} \mathrm{C}$ of $T_{\text {amb }}$ ) in the LWIR. The resulting temperature differences are far less than those for ozone. Consequently, the transmittancebased approximation is largely correct outside the LWIR window where the atmosphere is opaque, and shows a lower underestimation of $P_{\text {cooling }}$ than seen for ozone at individual LWIR wavelengths (Figure 1B). However, unlike for ozone, the underestimation occurs over a much broader bandwidth across the LWIR, and for dry atmospheres, across the $16-20 \mu \mathrm{m}$ wavelengths, which adds to a significant total when integrated. 

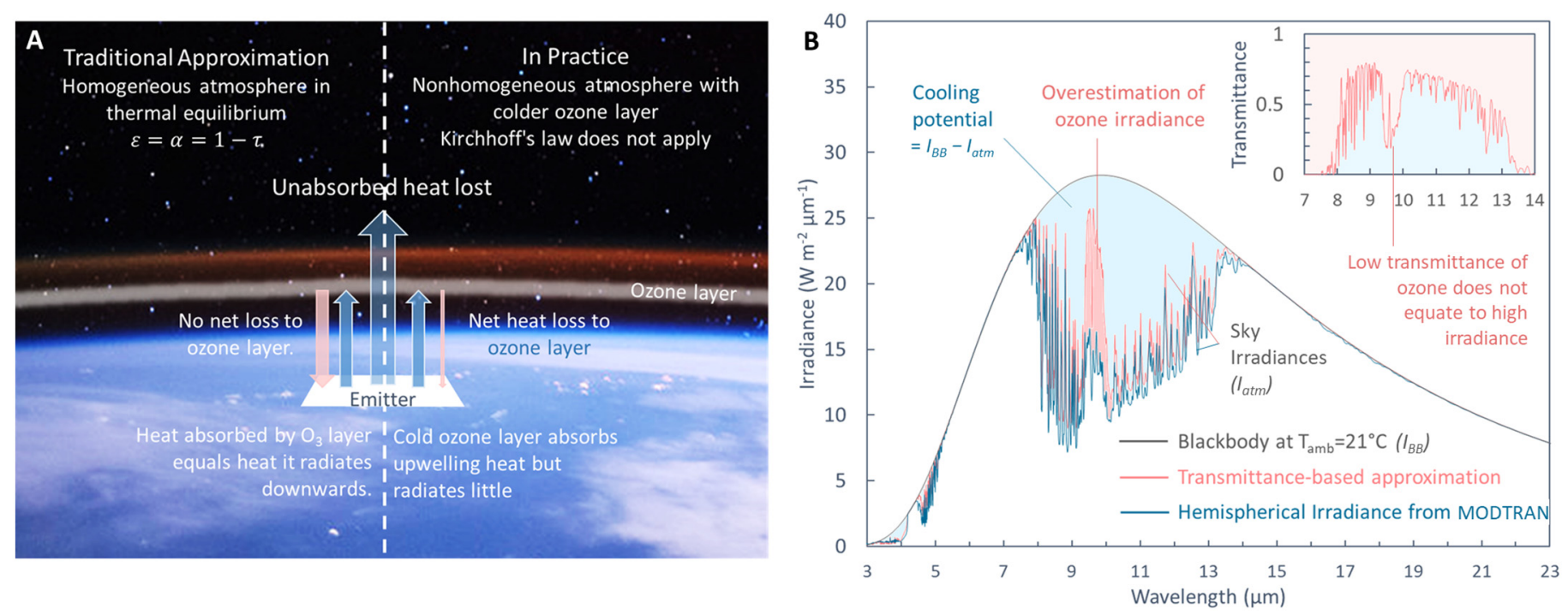

Figure 1. (A) Conceptual framework for traditional atmospheric thermal radiation against real case atmospheric thermal radiation. The photograph in the background was taken by NASA [31], and used under the Creative Commons-CC-BY-NCND 2.0 License. (B) The spectral hemispherical atmospheric irradiance from the transmittance-based approximation and that from MODTRAN.

It should be noted that Granqvist explicitly proposed the transmittance-based cosine approximation for use with a box model for calculating the radiative cooling powers of $\mathrm{SiO}$ films on metal. Since the irradiance from ozone and absorptance/emittance of SiO films have little overlap and the $\mathrm{SiO}$ film has a narrowband emittance, such a choice is justifiable in that context. However, the approximation has since been used to calculate the radiative cooling potentials of ideal emitters and cooling powers of radiative coolers with different spectral emittances, leading to both a systematic underestimation of cooling potential and a related overestimation of performance [14-29]. The MODTRAN hemispherical emittance, which is more accurate, should ideally be used instead. Indeed, Granqvist used a similar model in a later work [5]. However, because publicly available versions of MODTRAN contain no angle resolved information [30,32] to date, it has mostly been used for ideal emitters or real ones with ultra-wide angle emittances [33]. Furthermore, given the widespread use of the transmittance-based model in the radiative cooling community, it may be expedient for researchers to employ a modified version of the model that provides the flexibility needed to accurately model spectrally and angularly selective radiative coolers. Towards that end, in the subsequent section, we propose a correction to the transmittance-based cosine approximation that reduces the systematic overestimation of the ozone's irradiance while retaining the necessarily angular resolution of the model.

\section{Temperature-Corrections of the Transmittance-Based Model}

Although the publicly accessible MODTRAN hemispherical irradiance model provides a highly accurate estimate of the cooling potential, it does not contain angle-resolved information, which is crucial for calculating the performance of typical radiative coolers whose emittances can vary considerably with angle $[1,15,34,35]$, and which the transmittance-based cosine approximation provides. Therefore, here, we propose a correction that retains the mathematical fundamentals of the original model, but corrects for the overestimation of the irradiance from greenhouse gases. To do so, the lower effective temperatures of the ozone layer and water vapor $+\mathrm{CO}_{2}$ in the LWIR, which allow for heat loss from the terrestrial environment, must be taken into account. The ozone layer is kilometers-thick, and $\mathrm{CO}_{2}$ and water vapor are distributed throughout the atmosphere, which means that their radiative contributions are determined by a temperature distribution along their height. However, we can simplify calculations assuming that the irradiance of the ith gaseous component arises from a specific combination of its emittance $\varepsilon_{i}$ and effective temperature $T_{i}$. The 
irradiance $I_{a t m}$ can then be separated into two contributions: one for ozone, which is distributed high in the atmosphere, and one for $\mathrm{CO}_{2}+$ water vapor, which is distributed throughout, as follows:

$$
I_{\text {atm }}(\theta, \lambda)=\varepsilon_{\text {ozone }}(\theta, \lambda) \times I_{B B}\left(T_{\text {ozone }}, \lambda\right)+\varepsilon_{\text {rest }}(\theta, \lambda) \times I_{B B}\left(T_{\text {rest }}, \lambda\right)
$$

The directional emittances $\varepsilon_{i}(\theta, \lambda)$ of each component are calculated using Equations (2)-(4), using their transmittance instead of $\tau_{a t m}$. The transmittance $\tau_{\text {rest }}$ of water vapor $+\mathrm{CO}_{2}$, which effectively occur below the ozone layer, is calculated using MODTRAN by setting the atmospheric ozone concentration to zero. The transmittance of ozone is calculated as follows:

$$
\tau_{\text {Ozone }}(\theta, \lambda)=\tau_{\text {atm }}(\theta, \lambda) / \tau_{\text {rest }}(\theta, \lambda)
$$

As mentioned earlier, for the transmittance-based cosine model, it is reasonable to assume that the effective temperature of water vapor $+\mathrm{CO}_{2}$ where it is highly absorptive is the ambient temperature $T_{a m b}$ and the effective temperature of the completely masked ozone layer beyond is $0 \mathrm{~K}$. It thus remains to calculate the effective temperature $T_{\mathrm{Ozone}}$ of the ozone layer in the LWIR and $T_{\text {rest }}$ of water vapor and $\mathrm{CO}_{2}$ in the LWIR within the 16-20 $\mu \mathrm{m}$ range. To do so, we first obtain, from MODTRAN, the effective hemispherical irradiance of $\mathrm{CO}_{2}$ and water vapor $I_{r e s t}$ (i.e., without ozone) and that of the ozone layer $I_{\text {Ozone }}$ by subtracting $I_{\text {rest }}$ from the hemispherical irradiance of the whole atmosphere $\left(I_{\text {atm }}\right)$. In parallel, we also calculate the hemispherical emittance of water vapor $+\mathrm{CO}_{2}$, and the ozone layer from the directional values $\varepsilon_{\text {rest }}(\lambda, \theta)$ and $\varepsilon_{\text {Ozone }}(\lambda, \theta)$ calculated earlier. We then solve for $T_{i}(\lambda)$ using the equation:

$$
I_{i}(\lambda)=\varepsilon_{i}(\lambda) \int_{0}^{2 \pi} \int_{0}^{\frac{\pi}{2}} 2 h c^{2} \lambda^{-5}\left(e^{\frac{h c}{\lambda k_{B} T_{i}(\lambda)}}-1\right)^{-1} \cos \theta \sin \theta d \theta d \varphi
$$

Plots of $T_{\text {rest }}$ and $T_{\text {Ozone }}$ are presented in Figure 2 for the six MODTRAN standard atmospheres: US standard, Tropical, Midlatitude summer, Midlatitude winter, Subarctic summer and Subarctic winter. As shown, the effective temperatures of the gaseous components are drastically lower than $T_{a m b}$, with $\Delta \mathrm{T}$ (lambda) being $50-100{ }^{\circ} \mathrm{C}$ for ozone and $5-20{ }^{\circ} \mathrm{C}$ for water vapor $+\mathrm{CO}_{2}$, which the traditional transmittance-based model does not capture.

Equation (5), along with Equations (2)-(4), yields the directional irradiance, which in turn can be used to calculate the hemispherical irradiance $\left(I_{\text {atm }}\right)$ that corrects the overestimation of the transmittance-based model. As an illustration, we present the resulting hemispherical sky irradiances $I_{a t m}$ for the six MODTRAN standard atmospheres against the respective MODTRAN and transmission model outputs (Figure 3). As expected, the corrected transmission-based $I_{\text {atm }}$ is far closer to the MODTRAN irradiance than the original transmission-based approximation. More importantly, our method maintains its closeness to the MODTRAN model when the temperature $T_{a m b}$ is changed. Figure 4 shows the cooling potential $P_{\text {cooling }}=I_{B B}\left(T_{a m b}\right)-I_{a t m}$ of the traditional transmission-based model, the corrected model we propose and the MODTRAN irradiances of versions of the standard atmospheres at different temperatures. The values of $I_{a t m}$ are calculated using $T_{a m b}$ for the traditional transmission-based model, using $T_{a m b}$ and $\Delta \mathrm{T}$ in Figure 2 (assuming that $\Delta \mathrm{T}$ is unaffected by $T_{a m b}$ ) for the corrected model, and by scaling the irradiances of the standard atmosphere by the black-body spectra corresponding to $T_{a m b}$ for the MODTRAN model. As shown in Figure 4, relative to the MODTRAN model, the traditional approximation underestimates the cooling potential of radiative coolers by 6 to $24 \mathrm{Wm}^{-2}$ or 12 to $29 \%$ depending on the temperature and atmosphere type. The underestimations are large, particularly for high values of $I_{a t m}$. Our corrected model, by comparison, is within $1-8 \mathrm{Wm}^{-2}$ or $0.1-7 \%$ of the MODTRAN model, irrespective of the temperature, and the different total precipitable water and other greenhouse gas levels represented by the 
different atmospheres. The corrected transmission-based model thus provides an accurate irradiance relative to MODTRAN, while also providing angle-resolved irradiance values that the traditional transmission-based model provides.
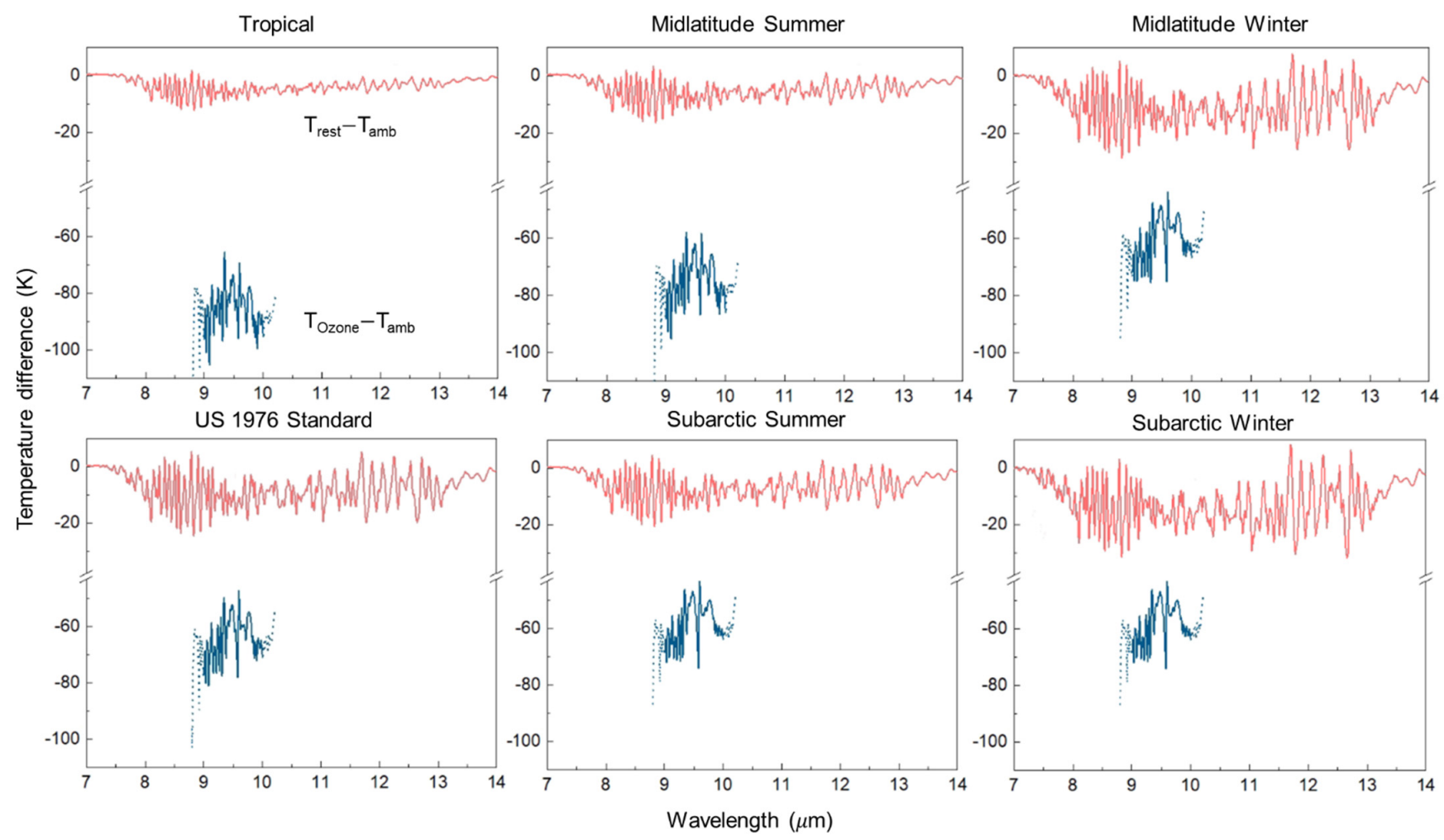

Figure 2. $\Delta T_{\text {ozone }}$ and $\Delta T_{\text {rest }}$ for the six MODTRAN standard atmospheres: Tropical, Midlatitude summer, Midlatitude winter, US 1976 Standard Atmosphere, Subarctic summer, Subarctic winter.
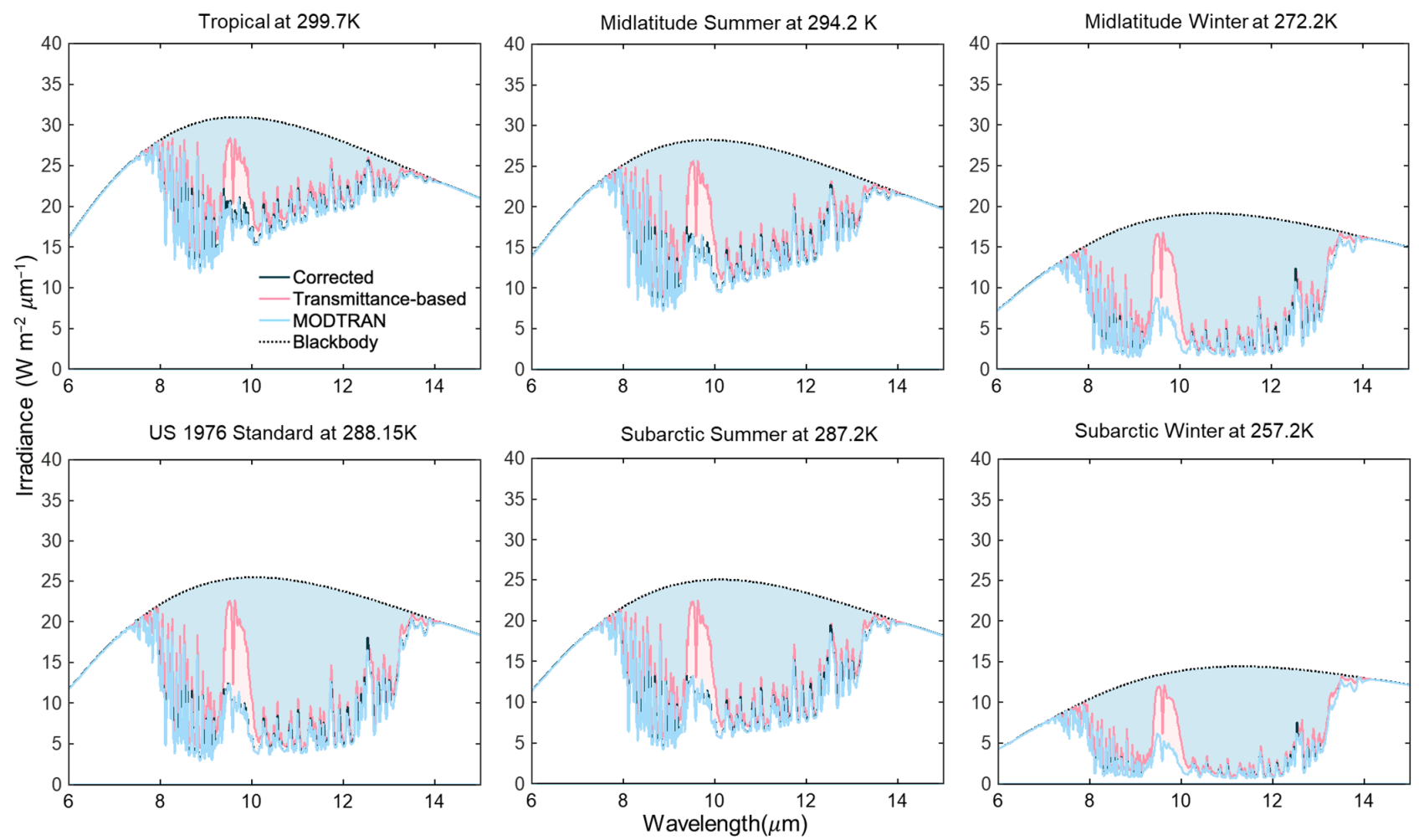

Figure 3. Spectral hemispherical atmospheric irradiance $I_{\text {atm }}$ for the transmittance-based model and our corrected model for the standard MODTRAN atmospheres. 

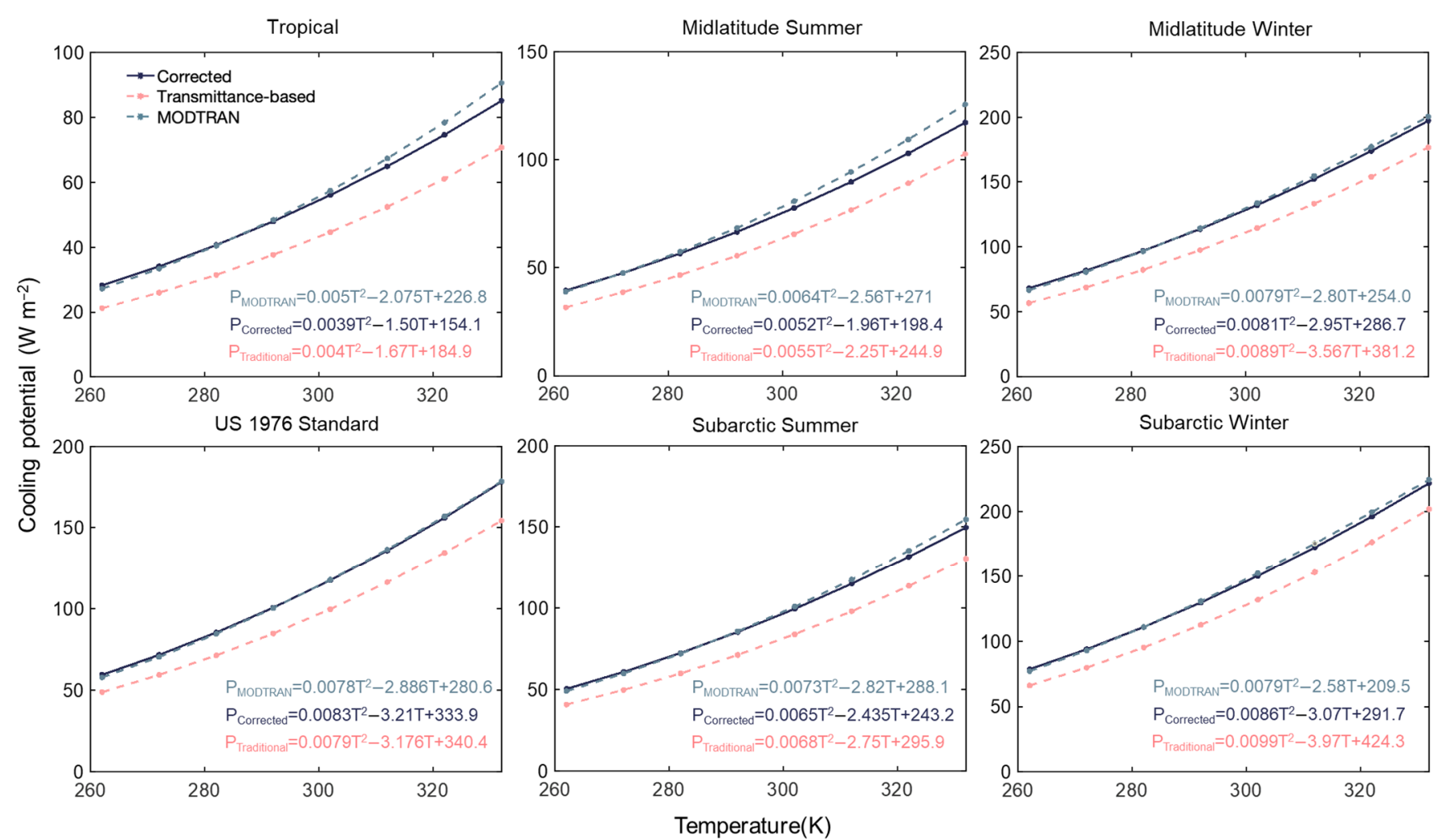

Figure 4. Maximum cooling potential $\left(P_{\text {cooling }}=I_{B B}\left(T_{a m b}\right)-I_{a t m}\right)$ calculated using the traditional transmittance-based model, the corrected model and MODTRAN for the six standard atmospheric profiles, but with $T_{a m b}$ varied from 262 to $332 \mathrm{~K}$. Dots indicate the calculated data, and the lines indicate fits with $\mathrm{R}^{2}>0.99$. Equations for the fits are provided. The data at low temperatures for the Tropical, Midlatitude Summer and Subarctic summer atmospheric profiles should be used with caution because at such temperatures, the atmosphere does not typically hold the high TPW levels that characterize the standard cases.

It should be noted that the transmittance-based model, and the correction we propose, are intended for clear, cloudless skies. This is sufficient for validating experimental performances of radiative coolers, as such experiments are usually performed under clear skies. However, the effects of clouds are important as well, particularly for mapping global average radiative cooling potentials. We note here that our model can, in principle, be modified to account for clouds - which are essentially finite layers of condensed water vapor with high absorption across the thermal and LWIR wavelengths-similar to how it accounted for the ozone layer in Equation (5). Doing so requires information about the thickness and type of clouds (e.g., thin cirrus vs. thick cumulus), their heights (which could be estimated from the dew point temperatures and atmospheric lapse rates), their coverage and view angle from the perspective of the radiative cooler. However, such a modification is beyond the scope of the present study, since the angle-resolved and spectrally resolved irradiances, which are the unique yields of the transmittance-based model, vary continuously as clouds move. In this case, the hemispherical irradiance becomes more important, and models which output it may be better suited. A number of such models, which take into account factors such as cloud cover, topography and altitude, exist in the literature $[6,8,10-12,36,37]$ and could be used in conjunction with satellite imagery of cloud covers and their calculated transmittances [38] as well as radiometric characterization of clouds [39,40] to calculate downwelling atmospheric irradiances. It should be noted that if the cloud cover is uniform and near-full, the motion of individual clouds has little impact and they behave as a layer, in which case, our model can be conveniently used. 


\section{Conclusions}

Two results stand out from our analysis. From a computational standpoint, our results indicate that a higher cooling power can be achieved by radiative coolers than is calculated using the traditional transmittance-based model under clear skies. This adds to their promise for cooling at local and global scales, with $\sim 20 \%$ greater cooling potentials possible for some atmospheres. However, our results also suggest that, except in rare cases, experimentally demonstrated radiative coolers perform less well relative to what is theoretically possible than usually thought. One reason for this could be that such radiative coolers often have their near-normal emittances reported and used in radiative cooling calculations rather than their true hemispherical emittance, which may require full angularly resolved measurements. Since hemispherical emittances are usually considerably lower than the near-normal emittances typically reported [41], this could explain why this mismatch has not been previously noted.

Given the above implications of our work, we believe that it may be useful to contextualize prior works that used the transmittance-based model, and that future works should account for the underestimation of the theoretical radiative cooling potential by the traditional transmittance-based model. Towards this end, we have provided analytical expressions of the cooling potentials for the different MODTRAN atmospheres as a function of temperature, as well as analytical expressions of $\Delta P_{\text {cooling. }}$. between the MODTRAN, transmittance-based and corrected models, in Table 1. Additionally, we have also made numerical data publicly available for the zenith-ward transmittances and $\Delta T_{i}$ for different model atmospheres, which can be used with our method to calculate angular and hemispherical emittances $[42,43]$. We hope that these resources will be useful to researchers modelling atmospheric irradiances for radiative cooling applications.

Table 1. Analytical expressions of the $P_{\text {cooling }}$ corrections between the MODTRAN, transmittance-based and corrected models.

\begin{tabular}{ccccccc}
\hline $\boldsymbol{\Delta} \boldsymbol{P}_{\text {cooling }}$ & Tropical & $\begin{array}{c}\text { Midlatitude } \\
\text { Summer }\end{array}$ & $\begin{array}{c}\text { Midlatitude } \\
\text { Winter }\end{array}$ & $\begin{array}{c}\text { US 1976 } \\
\text { Standard }\end{array}$ & $\begin{array}{c}\text { Subarctic } \\
\text { Summer }\end{array}$ & $\begin{array}{c}\text { Subarctic } \\
\text { Winter }\end{array}$ \\
\hline $\mathrm{P}_{\text {MODTRAN }}$ & $0.0011 \mathrm{~T}^{2}-0.57 \mathrm{~T}$ & $0.0012 \mathrm{~T}^{2}-0.6 \mathrm{~T}$ & $-0.002 \mathrm{~T}^{2}+$ & $-0.0005 \mathrm{~T}^{2}+$ & $0.0008 \mathrm{~T}^{2}-$ & $-0.0007 \mathrm{~T}^{2}+$ \\
$\mathrm{P}_{\text {Corrected }}$ & +72.7 & +72.6 & $0.15 \mathrm{~T}-32.7$ & $0.32 \mathrm{~T}-53.32$ & $0.385 \mathrm{~T}+45$ & $0.49 \mathrm{~T}-82.2$ \\
\hline $\mathrm{P}_{\text {MODTRAN }}$ & $0.001 \mathrm{~T}^{2}-0.4 \mathrm{~T}+$ & $0.0009 \mathrm{~T}^{2}-$ & $-0.001 \mathrm{~T}^{2}+$ & $-0.0001 \mathrm{~T}^{2}+$ & $0.0005 \mathrm{~T}^{2}-$ & $-0.002 \mathrm{~T}^{2}+$ \\
$\mathrm{P}_{\text {Traditional }}$ & 41.9 & $0.31 \mathrm{~T}+26.1$ & $0.767 \mathrm{~T}-127.2$ & $0.29 \mathrm{~T}-59.8$ & $0.071 \mathrm{~T}-7.9$ & $1.39 \mathrm{~T}-214.8$ \\
\hline
\end{tabular}

Author Contributions: J.M. conceived and determined the mathematical methodology for this work. X.H. and J.M. performed the calculations. A.P.R. supervised the study. J.M., X.H. and A.P.R. wrote the manuscript. All authors have read and agreed to the published version of the manuscript.

Funding: J. M. was supported by Schmidt Science Fellows, in partnership with the Rhodes trust. We also acknowledge support of the UCLA Hellman Fellows Award.

Data Availability Statement: Data and resources relevant to this study can be found at https: / / github.com/Raman-Lab-UCLA (accessed on 31 July 2021) and https:/ /jyotirmoymandal.com/ radiative-cooling-resources/ (accessed on 31 July 2021).

Conflicts of Interest: The authors declare no conflict of interest.

\section{References}

1. Granqvist, C.G.; Hjortsberg, A. Radiative Cooling to Low Temperatures: General Considerations and Application to Selectively Emitting SiO Films. J. Appl. Phys. 1981, 52, 4205-4220. [CrossRef]

2. Mandal, J.; Yang, Y.; Yu, N.; Raman, A.P. Paints as a Scalable and Effective Radiative Cooling Technology for Buildings. Joule 2020, 4. [CrossRef]

3. Levinson, R.; Akbari, H. Potential Benefits of Cool Roofs on Commercial Buildings: Conserving Energy, Saving Money, and Reducing Emission of Greenhouse Gases and Air Pollutants. Energy Effic. 2010, 3, 53-109. [CrossRef]

4. Munday, J.N. Tackling Climate Change through Radiative Cooling. Joule 2019, 3, 2057-2060. [CrossRef]

5. Eriksson, T.S.; Granqvist, C.G. Radiative Cooling Computed for Model Atmospheres. Appl. Opt. 1982, 21, 4381-4388. [CrossRef] 
6. Martin, M.; Berdahl, P. Characteristics of Infrared Sky Radiation in the United States. Sol. Energy 1984, 33, 321-336. [CrossRef]

7. Berdahl, P.; Fromberg, R. The Thermal Radiance of Clear Skies. Sol. Energy 1982, 29, 299-314. [CrossRef]

8. Li, M.; Peterson, H.B.; Coimbra, C.F.M. Radiative Cooling Resource Maps for the Contiguous United States. J. Renew. Sustain. Energy 2019, 11, 036501. [CrossRef]

9. Berger, X.; Buriot, D.; Garnier, F. About the Equivalent Radiative Temperature for Clear Skies. Sol. Energy 1984, 32, 725-733. [CrossRef]

10. Centeno, M. New Formulae for the Equivalent Night Sky Emissivity. Sol. Energy 1982, 28, 489-498. [CrossRef]

11. Crawford, T.M.; Duchon, C.E. An Improved Parameterization for Estimating Effective Atmospheric Emissivity for Use in Calculating Daytime Downwelling Longwave Radiation. J. Appl. Meteorol. Climatol. 1999, 38, 474-480. [CrossRef]

12. Iziomon, M.G.; Mayer, H.; Matzarakis, A. Downward Atmospheric Longwave Irradiance under Clear and Cloudy Skies: Measurement and Parameterization. J. Atmos. Solar Terr. Phys. 2003, 65, 1107-1116. [CrossRef]

13. Raman, A.P.; Anoma, M.A.; Zhu, L.; Rephaeli, E.; Fan, S. Passive Radiative Cooling below Ambient Air Temperature under Direct Sunlight. Nature 2014, 515, 540-544. [CrossRef] [PubMed]

14. Rephaeli, E.; Raman, A.; Fan, S. Ultrabroadband Photonic Structures to Achieve High-Performance Daytime Radiative Cooling. Nano Lett. 2013, 13, 1457-1461. [CrossRef]

15. Gentle, A.R.; Smith, G.B. A Subambient Open Roof Surface under the Mid-Summer Sun. Adv. Sci. 2015, 2, 1500119. [CrossRef]

16. Hossain, M.M.; Jia, B.; Gu, M. A Metamaterial Emitter for Highly Efficient Radiative Cooling. Adv. Opt. Mater. 2015, 3, 1047-1051. [CrossRef]

17. Hossain, M.M.; Gu, M. Radiative Cooling: Principles, Progress, and Potentials. Adv. Sci. 2016, 3, 1500360. [CrossRef]

18. Zhao, D.; Aili, A.; Zhai, Y.; Xu, S.; Tan, G.; Yin, X.; Yang, R. Radiative Sky Cooling: Fundamental Principles, Materials, and Applications. Appl. Phys. Rev. 2019, 6, 021306. [CrossRef]

19. Aili, A.; Zhao, D.; Lu, J.; Zhai, Y.; Yin, X.; Tan, G.; Yang, R. A KW-Scale, 24-Hour Continuously Operational, Radiative Sky Cooling System: Experimental Demonstration and Predictive Modeling. Energy Convers. Manag. 2019, 186, 586-596. [CrossRef]

20. Son, S.; Liu, Y.; Chae, D.; Lee, H. Cross-Linked Porous Polymeric Coating without a Metal-Reflective Layer for Sub-Ambient Radiative Cooling. ACS Appl. Mater. Interfaces 2020, 12, 57832-57839. [CrossRef]

21. Liu, C.; Wu, Y.; Wang, B.; Zhao, C.Y.; Bao, H. Effect of Atmospheric Water Vapor on Radiative Cooling Performance of Different Surfaces. Sol. Energy 2019, 183, 218-225. [CrossRef]

22. Zhu, Y.; Qian, H.; Yang, R.; Zhao, D. Radiative Sky Cooling Potential Maps of China Based on Atmospheric Spectral Emissivity. Sol. Energy 2021, 218, 195-210. [CrossRef]

23. Huang, W.; Chen, Y.; Luo, Y.; Mandal, J.; Li, W.; Chen, M.; Tsai, C.-C.; Shan, Z.; Yu, N.; Yang, Y. Scalable Aqueous Processing-Based Passive Daytime Radiative Cooling Coatings. Adv. Funct. Mater. 2021, 31, 2010334. [CrossRef]

24. Chen, M.; Yi, Z.; Tao, S.; Wang, S.; Fang, Z.; Lu, C.; Xu, Z. A Pragmatic Device Based on a Double-Sided Functional Structure for Efficient Water Harvesting. Glob. Challenges 2020, 4, 1900094. [CrossRef] [PubMed]

25. Kim, M.; Lee, D.; Son, S.; Yang, Y.; Lee, H.; Rho, J. Visibly Transparent Radiative Cooler under Direct Sunlight. Adv. Opt. Mater. 2021, 9, 2002226. [CrossRef]

26. Zhang, Z.; Dai, Y.; Ma, C. Design of Selectively Multilayered Periodic Gratings by PSO Algorithm for Radiative Cooling. Opt. Commun. 2021, 500, 127323. [CrossRef]

27. Xiang, B.; Zhang, R.; Luo, Y.; Zhang, S.; Xu, L.; Min, H.; Tang, S.; Meng, X. 3D Porous Polymer Film with Designed Pore Architecture and Auto-Deposited SiO2 for Highly Efficient Passive Radiative Cooling. Nano Energy 2021, 81, 105600. [CrossRef]

28. Zhou, K.; Li, W.; Patel, B.B.; Tao, R.; Chang, Y.; Fan, S.; Diao, Y.; Cai, L. Three-Dimensional Printable Nanoporous Polymer Matrix Composites for Daytime Radiative Cooling. Nano Lett. 2021, 21, 1493-1499. [CrossRef] [PubMed]

29. Zhu, R.; Hu, D.; Chen, Z.; Xu, X.; Zou, Y.; Wang, L.; Gu, Y. Plasmon-Enhanced Infrared Emission Approaching the Theoretical Limit of Radiative Cooling Ability. Nano Lett. 2020, 20, 6974-6980. [CrossRef]

30. Spectral Sciences Incorporated MODTRAN ${ }^{\circledR}$. Available online: http://modtran.spectral.com/modtran_home (accessed on 19 October 2019).

31. Johnson, N. The Atmospheric Glow and the Milky Way's Stars; NASA: Washington, DC, USA, 2020.

32. University of Chicago MODTRAN Infrared Light in the Atmosphere. Available online: http://climatemodels.uchicago.edu/ modtran/ (accessed on 5 May 2020).

33. Mandal, J.; Fu, Y.; Overvig, A.C.; Jia, M.; Sun, K.; Shi, N.N.; Zhou, H.; Xiao, X.; Yu, N.; Yang, Y. Hierarchically Porous Polymer Coatings for Highly Efficient Passive Daytime Radiative Cooling. Science 2018, 362, 315. [CrossRef] [PubMed]

34. Gentle, A.R.; Smith, G.B. Radiative Heat Pumping from the Earth Using Surface Phonon Resonant Nanoparticles. Nano Lett. 2010, 10, 373-379. [CrossRef]

35. Chen, Z.; Zhu, L.; Raman, A.; Fan, S. Radiative Cooling to Deep Sub-Freezing Temperatures through a 24-h Day-Night Cycle. Nat. Commun. 2016, 7, 1-5. [CrossRef]

36. Malek, E. Evaluation of Effective Atmospheric Emissivity and Parameterization of Cloud at Local Scale. Atmospheric Res. 1997, 45, 41-54. [CrossRef]

37. Aubinet, M. Longwave Sky Radiation Parametrizations. Sol. Energy 1994, 53, 147-154. [CrossRef]

38. Srivastava, A.; Rodriguez, J.F.; Saco, P.M.; Kumari, N.; Yetemen, O. Global Analysis of Atmospheric Transmissivity Using Cloud Cover, Aridity and Flux Network Datasets. Remote Sens. 2021, 13, 1716. [CrossRef] 
39. Hirsch, E.; Agassi, E.; Koren, I. A Novel Technique for Extracting Clouds Base Height Using Ground Based Imaging. Atmospheric Meas. Tech. 2011, 4, 117-130. [CrossRef]

40. Shaw, J.A.; Nugent, P.W. Physics Principles in Radiometric Infrared Imaging of Clouds in the Atmosphere. Eur. J. Phys. 2013, 34, S111-S121. [CrossRef]

41. Mandal, J. A Survey of Radiative Coolers in the Literature. Available online: https://engrxiv.org/nup6c/ (accessed on 31 July 2021).

42. Mandal, J. Resources for Radiative Cooling Research. Available online: https://jyotirmoymandal.com/radiative-coolingresources/ (accessed on 31 July 2021).

43. Raman, A.P. Raman Lab @ UCLA. Available online: https:/ / github.com/Raman-Lab-UCLA (accessed on 31 July 2021). 